
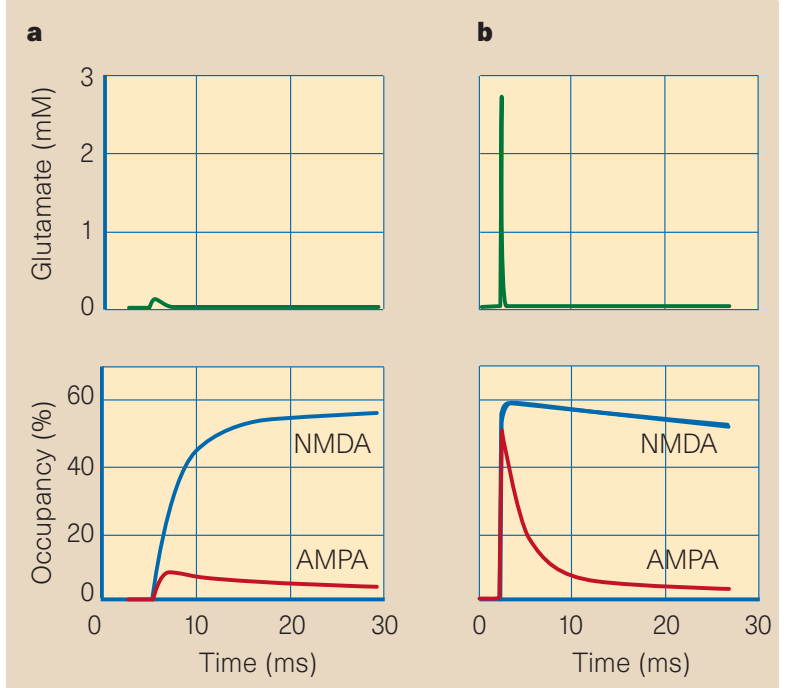

Figure 1 Occupancy of AMPA and NMDA receptors after release of the neurotransmitter glutamate. a, A relatively prolonged but lowconcentration pulse of glutamate leads to very low occupancy of AMPA receptors (defined as the proportion of doubly bound receptors). b, A much briefer, highconcentration pulse gives a much higher peak occupancy of AMPA receptors, approaching that of NMDA receptors. The two different glutamate pulses give similar peak occupancies of NMDA receptors $(\sim 56 \%)$. (For details of simulations see ref. 7 .) delivered in rapid succession should be no larger than that after one pulse — assuming that either or both stimuli gave rise to glutamate release. Transmission failures could be easily identified and excluded from the analysis. Contrary to expectation, the $\mathrm{Ca}^{2+}$ signal was consistently larger with two stimuli than with one. So, in some trials, glutamate must have been released in response to both stimuli, allowing more of the NMDA receptors to bind glutamate than when only one stimulus was delivered. In fact, after a single release, Mainen et al. estimate that the receptors were less than $60 \%$ occupied.

If NMDA receptors are incompletely occupied after transmitter release, how do AMPA receptors respond at all, given that their affinity for glutamate is so much lower? Should we expect to see synaptic events mediated by NMDA but not AMPA receptors? Synaptic signals mediated exclusively by NMDA receptors are, in fact, readily seen at the sub-physiological temperatures under which most in vitro experiments are done. And the finding that AMPA receptors often fail to detect transmitter release under these conditions has prompted the term 'silent synapses'. But at near-physiological temperatures - the conditions monitored by Mainen et al. — such silent synapses are rarely observed unless glutamate transporters are blocked $^{10}$. This result implies that glutamate uptake has a critical temperaturedependent role in determining receptor occupancy $^{3}$.

How might the speed at which glutamate is cleared explain how both NMDA and AMPA receptors contribute to synaptic signalling at physiological temperatures? The answer probably lies with the detailed kinetic properties of the two types of receptor. The NMDA receptors are less sensitive to glutamate when it is present for only a very short time than when it is present for a longer time. Indeed, by simulating the response of the two receptors to a very brief pulse - as may occur after exocytosis in vivo - the peak occupancies of both receptors turn out to be very similar (Fig. 1). This means that both NMDA and AMPA receptors may detect transmitter release to roughly the same extent. At sub-physiological temperatures, on the other hand, glutamate persists for longer owing, in part, to slower uptake, and NMDA receptors become relatively more sensitive to transmitter release than AMPA receptors. To test this hypothesis, we need more studies into the release, diffusion and uptake of glutamate in the extracellular space, and a detailed comparison of glutamate-receptor kinetics at different temperatures.

Whatever the outcome of the debate over the relative occupancy of AMPA and NMDA receptors, the striking conclusion of Mainen and colleagues' work is that a single release event falls roughly in the middle of the dynamic (response) range for a cluster of NMDA receptors. The lack of saturation is surprising, because it should make synapses less reliable than previously thought. On the other hand, the phenomenon illustrates a ubiquitous biological principle — that input is matched to output. This arrangement maximizes the sensitivity of the postsynaptic neuron to changes in the amount of glutamate released.

Dimitri M. Kullmann is in the Department of Clinical Neurology, Institute of Neurology, Queen

Square, University College London,

London WC1N 3BG, UK.

e-mail:dkullman@ion.ucl.ac.uk

1. Mainen, Z. F., Malinow, R. \& Svoboda, K. Nature 399, 151-155 (1999).

2. Clements, J. D. Trends Neurosci. 19, 163-171 (1996).

3. Tong, G. \& Jahr, C. E. Neuron 13, 1195-1203 (1994).

4. Silver, R. A., Cull-Candy, S. G. \& Takahashi, T. J. Physiol. (Lond.) 494, 231-250 (1996).

5. Liu, G., Choi, S. \& Tsien, R. W. Neuron 22, 395-409 (1999).

6. Patneau, D. K. \& Mayer, M. L. J. Neurosci. 10, 2385-2399 (1990).

Rusakov, D. A. \& Kullmann, D. M. J. Neurosci. 18, 3158-3170 (1998).

8. Svoboda, K. \& Mainen, Z. F. Neuron 22, 427-430 (1999).

9. Lester, R. A. et al. Nature 346, 565-567 (1990).

10. Asztely, F., Erdemli, G. \& Kullmann, D. M. Neuron 18, 281-293 (1997).

^๑) 1999 Macmillan Magazines Ltd
Daedalus

\section{Cultured diamonds}

One of the best ways of purifying a chemical substance is by crystallization. A growing crystal accepts molecules that fit its lattice, and rejects those that do not, so a pure product will crystallize from even quite an impure solution. Indeed, even thermodyamically unstable crystals will grow from a solution, if a few 'seed crystals' are added to give them a start.

The most valuable of all thermodynamically unstable crystals is, of course, diamond. So Daedalus wants to use the crystallization principle to grow big, pure diamonds. His first idea was to dissolve buckminsterfullerene (the only soluble form of carbon) in benzene or liquid xenon, add a few seed crystals of diamond, and let the solution evaporate. But it seems unlikely that a complete $\mathrm{C}_{60}$ molecule would rearrange to a diamond lattice merely by colliding with a diamond surface. A smaller carbon unit is required.

The smallest stable carbon unit is the $\mathrm{C}_{2}$ radical. It is most easily generated by heating carbon in a vacuum. The hot surface slowly evaporates by emission of these radicals - which is why the old carbon-filament lamps had such a limited life. $\mathrm{A}_{2}$ radical hitting a diamond surface should combine with it to extend the diamond lattice. So a steady rain of such radicals should, by the crystallization principle, grow a diamond of any size from a small initial seed.

Daedalus's diamond-growing apparatus has a source of $\mathrm{C}_{2}$ radicals (a carbon filament or arc) surrounded by a heated precious-metal nozzle to direct them as a beam towards an array of seed diamonds on a cooled platform. The whole thing is in a vacuum chamber. The seed diamonds are first cleaned by argon-ion bombardment, to remove surface contamination and expose their pristine lattice surfaces; the $C_{2}$ beam is then turned on to grow them to the required size. By narrowing and steering the beam, and orienting the diamonds under it, they can be shaped as well.

Cheap diamond-growing will transform the industry. Diamond jewellery will lose chic and value, while industrial and scientific diamonds will proliferate in the form of cutting tools, optical windows, heat-sinks and so on. But Daedalus muses that nature may have got there first. Many 'carbon stars' shower carbon vapour out into the interstellar vacuum. If a few seed diamonds happen to be orbiting in the vicinity, they might over millions of years grow into diamond planets.

David Jones 